\title{
Data-Driven Analysis of Friction Stir Welding for Aerospace Applications
}

\author{
Marta CAMPS ${ }^{\mathrm{a}, \mathbf{1}}$, Maddi ETXEGARAI ${ }^{\mathrm{a}}$, Francesc BONADA ${ }^{\mathrm{a}}$, William LACHENY ${ }^{\mathrm{b}}$, \\ Dorick BALLAT-DURAND ${ }^{\mathrm{b}}$, Sylvain PAULEAU ${ }^{\mathrm{b}}$ and Xavier DOMINGO ${ }^{\mathrm{a}}$ \\ ${ }^{a}$ Eurecat, Centre Tecnologic de Catalunya, Unit of Applied Artificial Intelligence, Av. \\ Carrer de Bilbao, 72, 08005 Barcelona, Spain \\ ${ }^{b}$ Ariane Group, 51/61 route de Verneuil, Bâtiment 71 - Bureau 142, 78131 - Les \\ Mureaux - France
}

\begin{abstract}
Industry 4.0 and the digitalization of the manufacturing processes have brought new opportunities and strategies for process control and optimization. Friction Stir Welding is becoming a relevant manufacturing technology for several applications, among them the aerospace sector. This work presents the first data analysis and characterization of the Friction Stir Welding process of the Pre-Final Assembly Line of the new Ariane 6 launcher. Process monitoring data is captured and analyzed to provide predictive quality solutions for improving manufacturing key performance indicators and bring smart manufacturing and Industry 4.0 digitalization into the aerospace manufacturing sector. The results show promising performance for abnormal behavior detection, leveraging on a tailored data manipulation approach for this unique use case.
\end{abstract}

Keywords.Feature Engineering, Machine Learning, Predictive Quality, Industry 4.0

\section{Introduction}

The proliferation of digitalization in manufacturing processes, fostered by Industry 4.0, has brought a new set of functionalities [1] with the potential to transform production by reinventing the monitorization, control and optimization of the whole system[2]. This new scenario is characterized by larger amounts of process data. Thus, data-driven solutions can provide major benefits in complex manufacturing processes [3-4].

As part of the H2020 SESAME project ${ }^{2}$, the Friction Stir Welding (FSW) station of Pre-Final Assembly Line of the new Ariane 6 launcher is monitored. FSW [5] is raising as a key manufacturing technology in different high added value sectors. Emerging research is being carried out to characterize and understand the importance of the process parameters of the FSW process by means of Machine Learning (ML) approaches[5-6]. SESAME use case goes one step further due to the quality excellence required for the aerospace industry. Data-driven methods that ensure continuous quality estimation at each step of the process will bring the aerospace industry into the digital manufacturing paradigm, with a focus on the production Key Performance Indicators. In this scenario, process monitoring strategies that build upon ML can be applied to ensure quality estimation and zero-defect propagation along the assembly line. In this document, a preliminary analysis of the first experimental weldings is presented, as well as the

\footnotetext{
${ }^{1}$ Marta Camps, Eurecat, Centre Tecnològic de Catalunya, Applied Artificial Intelligence Dpt., Av. Carrer de Bilbao, 72, 08005 Barcelona, Spain; E-mail: marta.camps@eurecat.org.

2 www.sesame-space.eu
} 
convenience of the feature engineering layers to detect anomalies in the data that can be related to non-quality in the welded part.

\section{Experimental Data}

The Ariane 6 Pre-Final Assembly Line (P-FAL) is composed of two FSW stations, in charge of the longitudinal and circular weldings of the panels of the tanks. In this work, data from two different production batches of the circular station is presented. For each welding test, the evolution of 56 process variables is recorded. Different magnitudes are gathered for the different axis and subsystems of the welding station, presenting a diverging behaviour needed to be considered. The data analyzed is part of a preliminary study to find the optimal functioning point of the FSW stations. In the future, weldings will be performed with stable parameters and will enable further quality analysis.

\section{Data segmentation: Regions of Interest}

The principal strategy to tune ML models to estimate the quality and performance of the welding is to segment the data gathered into representative sections that can be then compared. Due to the high complexity of the assembly line, it is difficult to relate the dataset to a specific section or point in the welded part.. We propose to segment the welding into representative parts, following the structure of the circular FSW station.

Identifying this sections will enable comparisions between different experiments and sections, while building a feature engineering layer to define a common ground. The Tool Holder Temperature parameter (Figure 1) shows a pattern that allows identifying 16 sections, based on temperature spikes produced during the welding process.

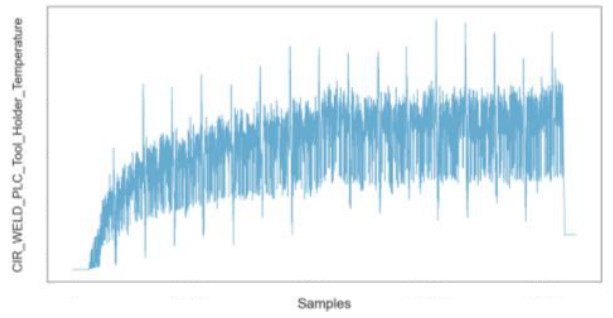

Figure 1. Welding dataset segmentation based on the Tool Holder Temperature behaviour.

\section{Feature Engineering}

To compare different experiments and different sections of the experiment, as they all differ in time duration and samples, equivalent variables must be set. To define a common ground and establish a fair comparison, the following set of features are proposed to capture the relevant information of each process parameter: min value, max value, mean value, standard deviation, number of samples, max value of the first order derivate, the two highest Power Spectral Density[7] and their corresponding frequencies. Comparing the proposed features for different experimental datasets and sections will allow identifying abnormal behaviors that can impact the welding quality. Figure 2 
shows features values for the Spindle Temperature as a function of the section (x-axis) and the dataset file (colour). The different behaviour of section 11 can be observed.

CIR_WELD_NC_SP_Temperature
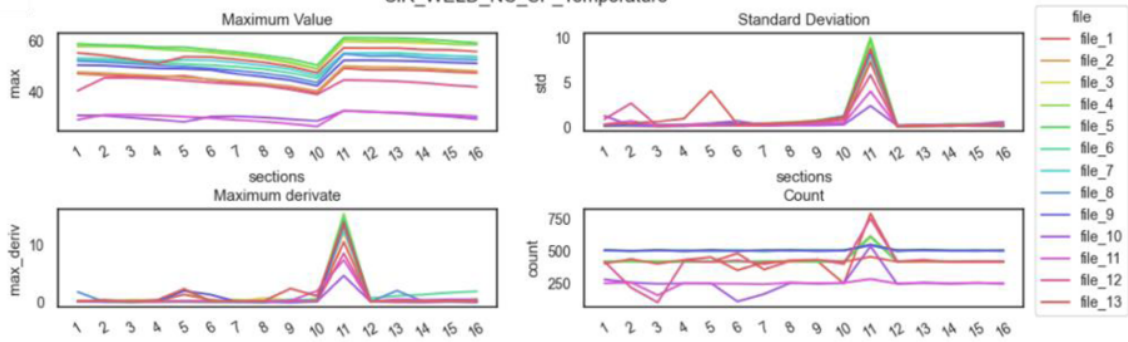

Figure 2. Example of features evolution for the Spindle Temperature parameter during the welding tests.

\section{Complexity reduction and cross decomposition}

Complexity reduction algorithms, such as Principal Component Analysis (PCA)[8], transform the problem by decreasing the data dimensionality, making the system more understandable for the process expert and encouraging their collaboration with ML systems.To compare different segments of the welding, a PCA projection is applied to the features. This analysis will help to identify clusters and abnormal behaviors. The presented results evaluate the different behavior of a given parameter among the 13 experiments and the 16 welding sections.
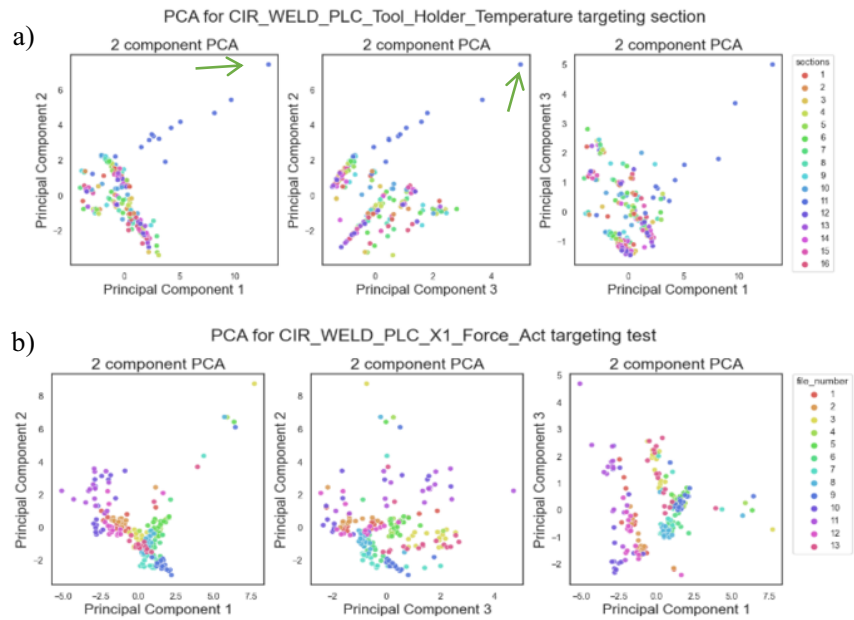

Figure 3. a) 3-PCA projection for the Tool Holder Temperature targeting welding sections. b) 3-PCA projection for the Force in $\mathrm{X} 1$ axis targeting experiments.

Figure 3a) presents the projection on the PCA space defined by sections for the Tool Holder Temperature, where the three components explain the $86,96 \%$ of data variance. It shows a clear cluster formed by section 11 . This cluster is related to the lower temperature values that can be observed in Figure 1. However, one of the experiments behaves abnormally in section 11 , as pointed by the green arrow. This abnormal behavior can affect the quality of the welding and provides valuable insight to the process expert to drive their quality inspection strategy of the welded tank. In Figure $3 b$ ) the three- 
component projection of the features of the Force in the X-axis can be seen. The selected components explain $83,45 \%$ of the information. Each color corresponds to a different welding and each dot a distinct section. Each weld creates separated clusters, indicating a strong dependency on the experiment. In addition, several outliers can be identified, most of them with higher values of the first and second principal components.

\section{Discussion, conclusions and next steps}

This paper presents the first results of the H2020 SESAME project regarding the datadriven predictive quality modules for the novel Friction Stir Welding station of the PFAL of Ariane 6 launcher. A first analysis of the experimental data is described, focusing on the data preparation layer that will enable the future developments of the predictive quality modules. A feature engineering layer where several new features are created is introduced as well as a strategy for regions of interest segmentation that will allow the comparison of particular experiments and sections. The preliminary results already show the feasibility to identify abnormal behaviors in the welding process. Next steps will consider the correlation of the presented feature engineering strategy with the destructive inspection quality data, to feed supervised ML solutions: a classifier to determine the type of defect present in the welding and a regressor to estimate the severity of the defect.

\section{Acknowledgment}

This work has been carried out in the framework of the SESAME project, which has received funding from the European Union's Horizon 2020 research and innovation programme under grant agreement No 821875. The content of this paper reflects only the author's view; the EU Commission/Agency is not responsible for any use that may be made of the information it contains.

\section{References}

[1] Zheng T, Ardolino M, Bacchetti A, Perona M. The applications of Industry 4.0 technologies in manufacturing context: a systematic literature review. Int. J. Prod. Res., 59:6, 1922-1954

[2] Reis MS, Gins G. Industrial Process Monitoring in the Big Data/Industry 4.0 Era: from Detection, to Diagnosis, to Prognosis. Processes. 2017; 5(3):35.

[3] Nieves J, Santos L, Bringas P G. Combination of machine-learning algorithms for fault prediction in high-precision foundries. Lect. Notes Comput. Sci., vol. 7447 LNCS, no. PART 2, pp. 56-70, 2012

[4] Susto G A, Schirru A, Pampuri S, McLoone S, Beghi A, "Machine Learning for Predictive Maintenance: A Multiple Classifier Approach," in IEEE Trans. Industr. Inform, vol. 11, no. 3, pp. 812-820, June 2015.

[5] Thomas W.M, Nicholas E.D, Friction stir welding for the transportation industries, Materials \& Design, vol 18, Is 4-6, pp 269-273, 1997

[6] Nadeau F, Thériault B, Gagné M-O. Machine learning models applied to friction stir welding defect index using multiple joint configurations and alloys. P I MECH ENG L-J MAT , 234(5), 752-765

[7] Du Y, Mukherjee T, DebRoy T. Conditions for void formation in friction stir welding from machine learning. npj Comput Mater 5, 68 (2019)

[8] P. Welch. The use of the fast Fourier transform for the estimation of power spectra: A method based on time averaging over short, modified periodograms, IEEE Trans. Audio Electroacoust. 2020. vol. 15, pp. 70-73, 1967.

[9] Pearson K. On Lines and Planes of Closest Fit to Systems of Points in Space. Philos. Mag. Lett. 1901. 2 (11): $559-572$ 